\title{
Concomitant Retrograde Coronary Venous Infusion of Basic Fibroblast Growth Factor Enhances Engraftment and Differentiation of Bone Marrow Mesenchymal Stem Cells for Cardiac Repair after Myocardial Infarction
}

\author{
Xiao Wang1, Lei Zhen¹, Huangtai Miao'1, Qiwei Sun², Ya Yang², Bin Que, Edmundo Patricio Lopes Lao1, \\ Xingxin $\mathrm{Wu}^{1}{ }^{1}$, Hongmei Ren ${ }^{1}$, Shutian Shi ${ }^{1}$, Wayne B. Lau ${ }^{3}$, Xinliang Ma1,3, Changsheng Ma ${ }^{4}$, Shaoping \\ $\mathrm{Nie}^{1 凶}$ \\ 1. Emergency \& Critical Care Center, Beijing Anzhen Hospital, Capital Medical University, and Beijing Institute of Heart, Lung, and Blood Vessel \\ Diseases, Beijing, China; \\ 2. Department of Ultrasound, Beijing Anzhen Hospital, Capital Medical University, Beijing, China. \\ 3. Department of Emergency Medicine, Thomas Jefferson University, Philadelphia, PA 19107, USA. \\ 4. Department of Cardiology, Beijing Anzhen Hospital, Capital Medical University, Beijing, China.
}

$\triangle$ Corresponding author: Shaoping Nie, MD, PhD. Emergency \& Critical Care Center, Beijing Anzhen Hospital, Capital Medical University, No. 2 Anzhen Road, Chaoyang District, Beijing 100029, China. Tel: 86-10-84005256 Fax: 86-10-64439768 E-mail: spnie@ccmu.edu.cn.

() 2015 Ivyspring International Publisher. Reproduction is permitted for personal, noncommercial use, provided that the article is in whole, unmodified, and properly cited See http:/ /ivyspring.com/terms for terms and conditions.

Received: 2015.01.16; Accepted: 2015.05.09; Published: 2015.06.08

\begin{abstract}
Aim: Basic fibroblast growth factor (bFGF) increases the migration and viability of bone marrow mesenchymal stem cells (MSCs) in vitro. Retrograde coronary venous infusion can provide both increased regional bFGF concentrations and homogeneous cell dissemination. We determined whether retrograde delivery of bFGF enhances the potency of transplanted MSCs for cardiac repair in a canine infarct model.

Methods and Results: Under hypoxic conditions, cellular migration was significantly increased in MSCs co-cultured with bFGF compared to vascular endothelial growth factor or insulin-like growth factor, and bFGF promoted MSCs differentiation into a cardiomyocyte phenotype. A canine infarct model was employed by coronary ligation. One week later, animals were subjected to retrograde infusion of combination bFGF $(200 \mathrm{ng} / \mathrm{mL})$ and MSCs $\left(1 \times 10^{8}\right.$ cells $)(n=5), M S C s\left(1 \times 10^{8}\right.$ cells, $n=5)$, bFGF ( $200 n g / m L, n=5)$, or placebo (phosphate-buffered saline, $n=3$ ). Four weeks after infusion, only the bFGF+MSCs therapy exhibited significantly increased left ventricular ejection fraction (LVEF) by echocardiography ( $p<0.01$ vs pre-infusion), and the treatment effect (delta LVEF) was greater in the bFGF+MSCs group compared to saline $(7.43 \pm 1.51 \%$ versus $-10.07 \pm 2.94 \% ; p<0.001)$. Morphologic analysis revealed an increased infarct wall thickness in the bFGF+MSCs group compared to all others $(p<0.05)$, accompanied by increased vascular density and reduced apoptosis. Immunofluorescence demonstrated increased cell engraftment and enhanced vascular differentiation in the bFGF+MSCs group compared to MSCs alone $(p<0.05)$.

Conclusions: Retrograde coronary venous bFGF infusion augments engraftment and differentiation capacity of transplanted MSCs, recovering cardiac function and preventing adverse remodeling. This novel combined treatment and delivery method is a promising strategy for cardiac repair after ischemic injury.
\end{abstract}

Key words: Cell transplantation; Growth factor; Mesenchymal stem cells; Myocardial infarction; Retrograde. 


\section{Introduction}

Stem-cell based therapy has emerged as a potential therapeutic modality for regeneration and repair of damaged myocardium after myocardial infarction (MI) [1]. Bone marrow mesenchymal stem cells (MSCs) are easily isolated and amplified, possessing multilineage potential and apparent immuno-privilege, and are therefore a promising candidate for cardiac regeneration therapy [2]. Numerous preclinical [3-5] and clinical studies [6, 7] have demonstrated the ability of MSCs to attenuate reverse remodeling and restore cardiac function following acute MI. The underlying mechanisms include direct differentiation of implanted cells into new cardiomyocytes and vascular cells [8], augmented paracrine signaling [9], and recruitment of endogenous stem cells [3]. However, recent studies demonstrated limited in vivo differentiation capacity due to poor engraftment and survival of transplanted MSCs within infarcted myocardium, resulting in modest therapeutic effects [10].

The efficiency of stem cell retention within injured myocardium is related to sufficient cellular extravasation and local growth factor gradients [11]. Basic fibroblast growth factor (bFGF) is a potent mitogen that can stimulate migration, proliferation, and differentiation in various stem cell types [12]. In particular, bFGF increased the migratory activity of MSCs via activation of the Akt/protein kinase B pathway [13]. The bFGF-transfected MSCs exhibited enhanced viability and anti-apoptotic properties under hypoxic conditions [14]. It is therefore plausible that bFGF administration after MI may augment MSCs migration and engraftment within injured tissue, thus improving the efficacy of MSCs transplantation.

Several approaches have been developed to achieve extensive stem cell delivery into the damaged heart $[15,16]$. Although direct intramyocardial injection allows targeted cell delivery to ischemic regions, this method may cause mechanical injury and subsequent acute inflammation, markedly reducing survival of engrafted cells [17]. Intracoronary administration is not ideal, as there is high risk of coronary embolism[18], as well as low myocardial stem cell retention in the face of antegrade blood flow [19]. In contrast, percutaneous retrograde coronary venous infusion represents an attractive alternative method because it is minimally invasive, reproducible, and has potential advantages of more uniform and higher rate of delivery [15]. This technique achieved a 2-fold increase in tissue binding of bFGF compared to intracoronary infusion [20]. Coronary vein route may also provide more homogenous cell dissemination across the myocardium [21, 22]. Heretofore, the effi- cacy of combination bFGF and MSCs via coronary vein administration has not been investigated.

We hypothesized retrograde coronary venous infusion may provide a high bFGF concentration, and augment MSCs implantation by increasing the engraftment and survival of transplanted cells. To test this hypothesis, we employed a canine infarct model, and determined the effects of bFGF upon MSCs engraftment and differentiation via coronary vein infusion, and assessed their combined efficacy in improving cardiac repair via morphologic indices and functional outcomes.

\section{Methods}

An expanded Methods section is available in the Supplementary Materials.

This study conformed to the Interdisciplinary Principles and Guidelines for the Use of Animals in Research, Testing, and Education by the New York Academy of Sciences, Ad Hoc Animal Research Committee. All protocols were approved by the Institutional Ethics Committee of Beijing Anzhen Hospital, Capital Medical University, China.

For this study, adult male mongrel dogs weighing 15-20 kg were subjected to MI via ligation of left anterior descending (LAD) coronary artery and diagonal branches. After one week, all surviving animals underwent retrograde infusion of $10 \mathrm{~mL}$ of one of the following: combination bFGF $(200 \mathrm{ng} / \mathrm{mL})$ and MSCs $\left(1 \times 10^{8}\right.$ cells), MSCs alone $\left(1 \times 10^{8}\right.$ cells $)$, bFGF alone $(200 \mathrm{ng} / \mathrm{mL}$ ), or placebo (phosphate-buffered saline). Transthoracic echocardiography was performed at baseline, 1 week after MI (before infusion), and 4 weeks after infusion (just prior to euthanasia). After echocardiographic study, animals were euthanized, and the hearts were excised for histologic analysis.

\section{In vitro migration and differentiation assays}

To assess the migration capacity of MSCs, a

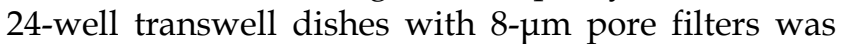
utilized. Cells were incubated with $50 \mathrm{ng} / \mathrm{mL}$ bFGF, $20 \mathrm{ng} / \mathrm{mL}$ vascular endothelial growth factor (VEGF), $2 \mathrm{ng} / \mathrm{mL}$ insulin-like growth factor (IGF-1), or medium alone. The number of migrated cells was counted via fluorescent microscopy. All experiments were tested under normoxic and hypoxic conditions.

To induce differentiation into a cardiomyocyte phenotype, $2 \times 10^{4}$ MSCs were incubated with or without $5 \mathrm{ng} / \mathrm{mL}$ bFGF. The differentiation capacity was verified by morphology changes and immunostaining for troponin I (TnI).

\section{Retrograde coronary venous infusion}

Retrograde coronary venous infusion was performed as previously described [21]. From LAD cor- 
onary angiography, the position of over-the-wire (OTW) balloon catheter was consistent with the occlusive site of the LAD (Figure 1). The balloon was inflated, and the selected perfusion medium was rapidly injected into the anterior interventricular vein (AIV). The balloon remained inflated for 10 minutes post injection to achieve maximum local delivery.

\section{Morphology and histology}

After echocardiographic study, cardiectomy was performed. Thereafter, the left ventricular (LV) myocardium was transversely sliced along the apical-basal axis. For morphologic analysis, infarct wall thickness was measured at the level of the papillary muscle, and calculated as a percentage of septal wall thickness. Hematoxylin and eosin, and Masson trichrome staining were performed as previously described. Fibrotic area was calculated by the proportion of collagen-stained areas to total tissue area. Blood vessel density was determined at the border zone of the myocardium by immunostaining with factor VIII-related antigen (FVIII) and a-smooth muscle actin (a-SMA). Apoptosis was detected with an in situ cell death detection kit per manufacturer protocol.

\section{Immunofluorescence}

The engraftment and differentiation of MSCs were evaluated in the MSCs $(n=3)$ and bFGF+MSCs $(n=3)$ groups. MSCs were labeled with enhanced green fluorescence protein (EGFP) and infused into the AIV. After four weeks, frozen sections $(7 \mu \mathrm{m})$ were incubated with antibodies against FVIII, a-SMA, TnI, and cardiac myosin heavy chain (MHC). The survival of engrafted cells was quantified in the infarct zone by counting EGFP-positive $\left(\mathrm{EGFP}^{+}\right)$cells by fluorescent microscopy. The differentiation capacity was evaluated based upon colocalization of EGFP with the endothelial cell marker FVIII, smooth muscle cell marker a-SMA, and cardiomyocyte marker TnI.

\section{Statistical analysis}

All values are presented as mean \pm SEM unless otherwise indicated. Statistical assessments were performed using SPSS 19.0 (SPSS Science, Chicago, IL). GraphPad Prism (version 5.0; GraphPad Software Inc, La Jolla, CA) was used to plot graphs. Data were first tested for normality (Kolmogorov-Smirnov test) and equality of variance (Levene's test). After confirmation of both normality and variance equality, comparisons between two groups were determined by unpaired Student $t$ test, and comparisons among multiple groups were made by 1-way or 2-way analysis of variance (ANOVA), followed by Tukey post hoc test when appropriate. The Welch correction was applied when unequal variances were identified. Intragroup comparisons at different time points were performed by paired Student $t$ test. Changes in echocardiography parameters over time among different groups were evaluated using 2-way repeated measures ANOVA with Bonferroni posttests when appropriate. A value of 2-tailed $p<0.05$ was considered statistically significant.
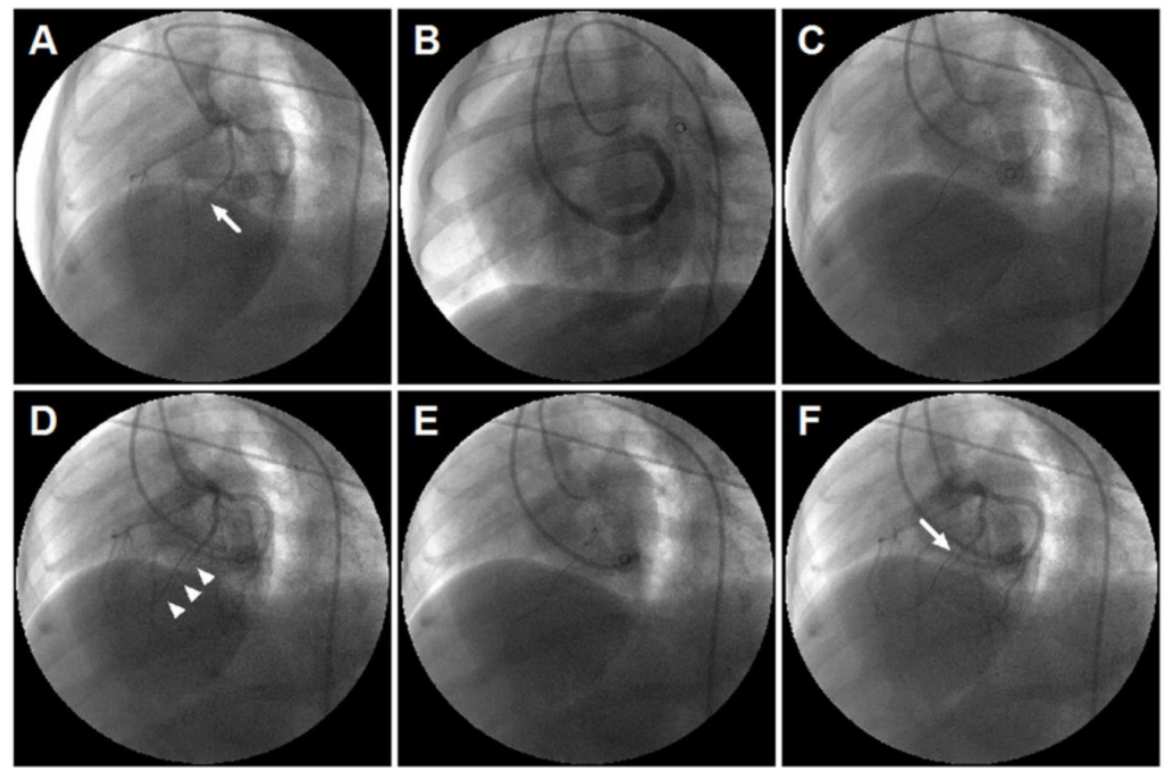

Figure 1. Retrograde coronary venous infusion. (A) Angiogram of the left coronary artery demonstrating occlusion of the left anterior descending (LAD) coronary artery (middle) (white arrow). (B) Retrograde venography of coronary sinus. (C) A 0.014-inch exchange-length extra support guide wire was advanced to the distal portion of anterior interventricular vein (AIV). (D) Angiogram of the left coronary artery demonstrating parallel guide wire travel to the LAD (white arrowheads). (E) An over-the-wire (OTW) balloon catheter $(2.5 \times 9 \mathrm{~mm}$ or $2.0 \times 9 \mathrm{~mm})$ was advanced and positioned in the mid-AIV. (F) Balloon inflation and simultaneous visualization of the left coronary artery showed that balloon position was consistent with the occlusive site of the LAD (white arrow). The delivery site was within the targeted infarct zone. 
A

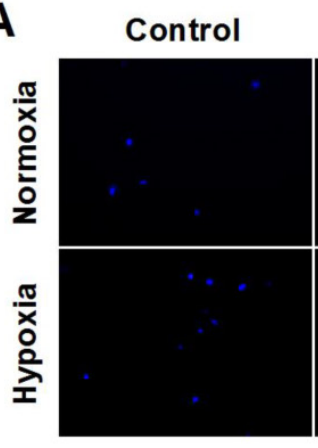

VEGF
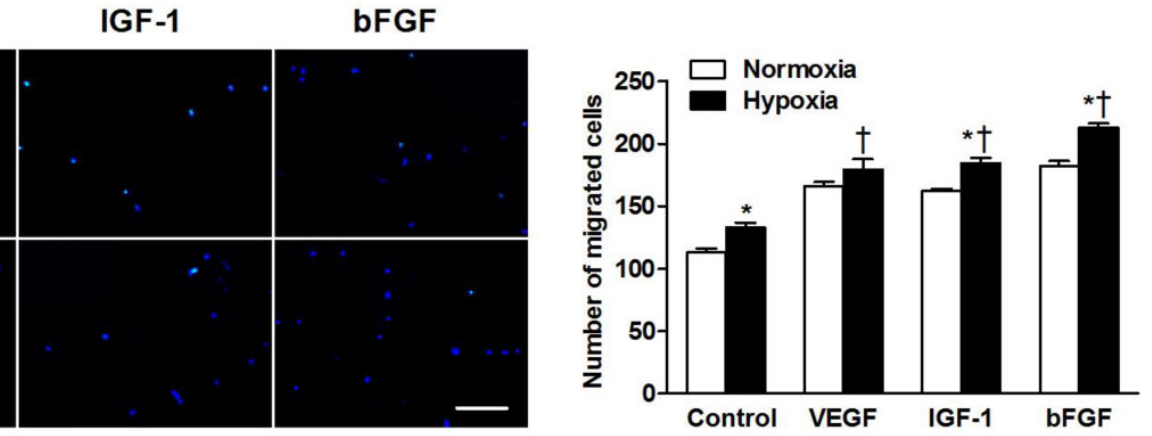

B

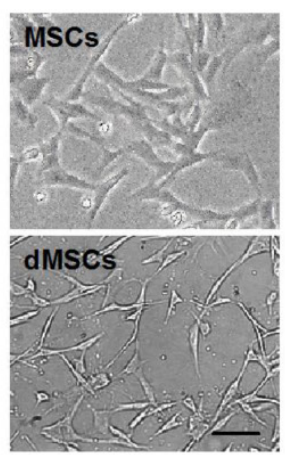

C
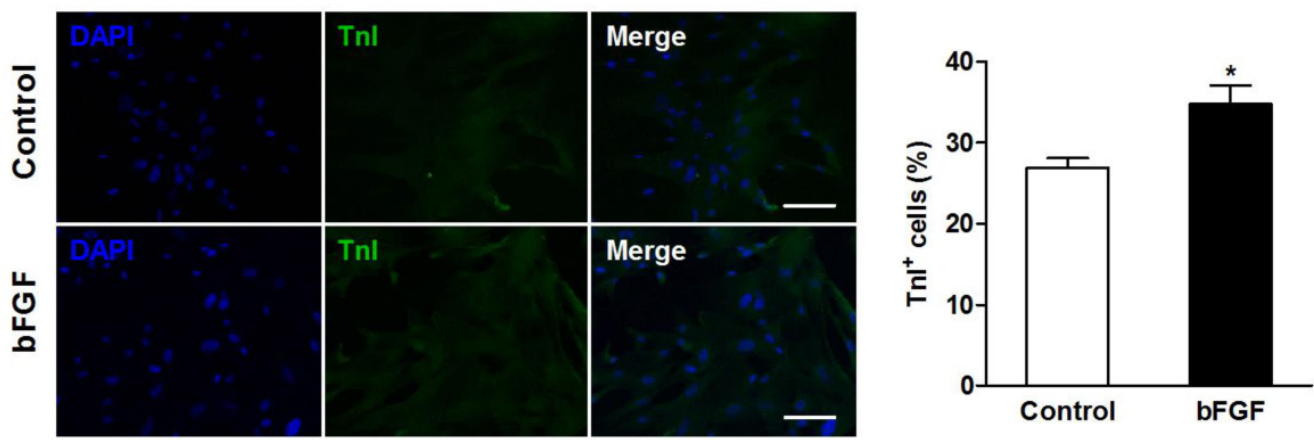

Figure 2. Basic fibroblast growth factor (bFGF) promotes mesenchymal stem cells (MSCs) migration and differentiation into a cardiomyocyte phenotype in vitro. (A) MSCs were cultured under normoxic and hypoxic conditions with $50 \mathrm{ng} / \mathrm{mL} \mathrm{bFGF}, 20 \mathrm{ng} / \mathrm{mL}$ vascular endothelial growth factor (VEGF), $2 \mathrm{ng} / \mathrm{mL}$ insulin-like growth factor (IGF-1), or control medium. Under hypoxic conditions, the number of migrated cells increased when incubated with VEGF, IGF-1, and bFGF. (B) Myotube-like structures were observed in differentiated MSCs (dMSCs). (C) Cotreatment with bFGF induced MSCs differentiation into a cardiomyocyte phenotype, as indicated by increased cardiac troponin I positive $\left(\mathrm{Tnl}^{+}\right)$cells. Nuclei were stained with 4,6-diamidino-2-phenylindole (DAPI). Scale bars: $200 \mu \mathrm{m}(\mathrm{A}) ; 100 \mu \mathrm{m}(\mathrm{B}$ and $\mathrm{C})$. Histograms represent mean $\pm \mathrm{SEM}$. A: $* p<0.05$ vs normoxia; $\nmid p<0.05$ vs control. All with 2 -way ANOVA. C: $* p<0.05$ vs control, unpaired Student $t$ test.

\section{Results}

\section{Study animals}

In total, 32 animals were subjected to MI. 7 animals had refractory ventricular fibrillation during surgery, and could not be resuscitated. 2 animals died at days 2 and 5 after infarction, likely due to congestive heart failure. Before infusion, 4 animals were excluded (3 with coronary vein anomalies precluding successful catheterization, and 1 with inadequate echocardiography imaging). The remaining 19 animals were randomly assigned to treatment with saline $(n=4)$, bFGF $(n=5)$, MSCs $(n=5)$, and bFGF+MSCs $(n=5)$. There were no instances of death, cardiac tamponade, or sustained arrhythmia during infusion. One animal in the saline group died 4 days post infusion. Finally, 18 animals (saline [ $n=3]$, bFGF $[n=5]$, MSCs [ $n=5]$, and bFGF+MSCs [ $=5]$ ) were included in the analysis.

\section{bFGF promotes MSCs migration and differen- tiation into a cardiomyocyte phenotype in vitro}

The number of migrated cells was significantly greater when incubated under hypoxic versus normoxic conditions in control $(p=0.006)$, IGF-1 $(p=0.002)$, and bFGF $(p<0.001)$ groups (Figure 2A). During hypoxia, MSCs exhibited increased migratory ability when cultured with VEGF, IGF-1, and bFGF (all $p<0.001$ vs control).

To confirm whether bFGF stimulates MSCs differentiation into a cardiomyocyte phenotype in vitro, we observed the morphology and cardiac-specific TnI expression of MSCs with or without bFGF. Differentiated MSCs showed myotube-like structure after 2 weeks (Figure 2B). The bFGF-treated MSCs expressed greater TnI expression. Quantitative analysis revealed that the number of the $\mathrm{TnI}^{+}$cells was significantly higher in the presence of bFGF versus control medium $(32.8 \pm 2.3 \%$ vs $27.0 \pm 1.2 \%$; $p=0.015$; Figure $2 \mathrm{C})$.

\section{Retrograde infusion of combining bFGF and MSCs improves cardiac function after $\mathrm{MI}$}

We tested whether bFGF might enhance the potential of transplanted MSCs for cardiac repair by serial echocardiographic analysis at baseline (before MI), 1 week after MI (before infusion), and 4 weeks after infusion (Table 1, Figure 3). The LV structure and function parameters at baseline and before infusion were comparable among the 4 groups $(p>0.05)$ (Table 1 , Figure 3A, 3C, and 3E). LV ejection fraction (LVEF) was improved in the bFGF+MSCs group by $11 \%$ $(p<0.01)$, but remained unchanged in the bFGF group 
$(p=0.480)$ and MSCs group $(p=0.124)$, and decreased in the saline group $(p<0.01)$ after 4 weeks (Figure $3 \mathrm{~A})$. The treatment effect (delta LVEF) was significantly greater in both the bFGF+MSCs group $(7.43 \pm 1.51 \%$ versus $-10.07 \pm 2.94 \% ; p<0.001)$ and MSCs group $(4.79 \pm 2.47 \%$ versus $-10.07 \pm 2.94 \% ; p<0.01)$ compared to saline, to greater degree in the combination group (Table 1, Figure 3B).

The left ventricular end-diastolic volume (LVEDV) increased in all groups (all $p<0.05$; Figure 3C), with modest attenuation in the intervention groups compared with saline group (ANOVA $p=0.053$; Table 1, Figure 3D). Furthermore, the left ventricular end-systolic volume (LVESV) remained stable in the intervention groups, but increased in the saline-treated group $(p<0.001$; Figure 3E). The absolute changes suggested attenuation of LVESV in the intervention groups (all $p<0.05$ vs saline), to greatest degree in the combination group (Table 1, Figure 3F).

Left ventricular end-diastolic diameter (LVEDD) and left ventricular end-systolic diameter (LVESD) demonstrated progressive chamber expansion in the saline group, a phenomenon not observed in the other treatment groups. All four groups exhibited decreased fractional shortening (FS) prior to treatment, without improvement after 4 weeks (Table 1).
A

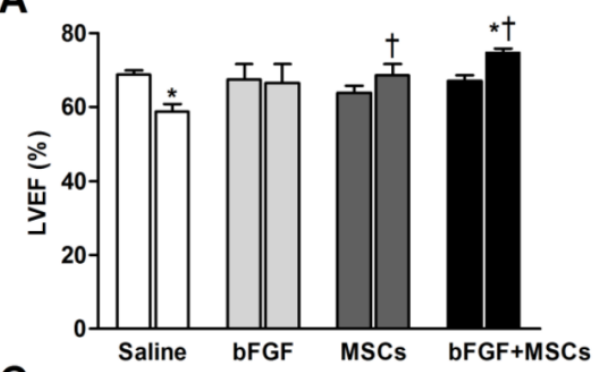

C

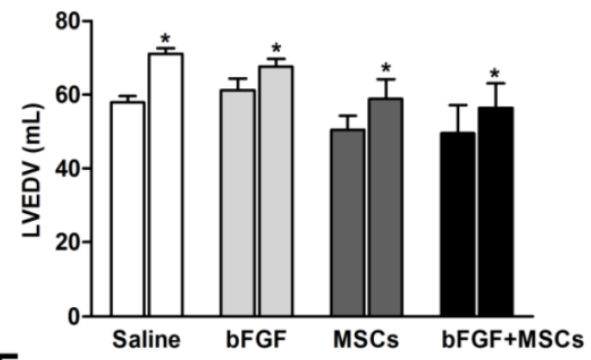

E

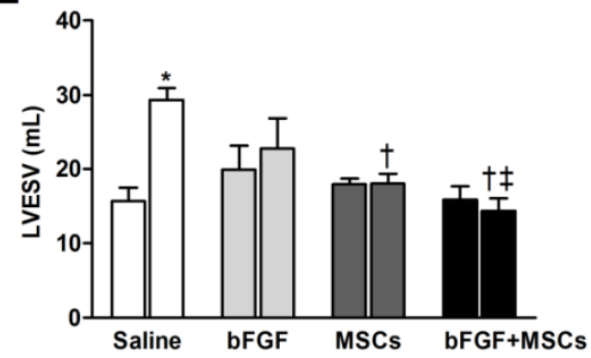

B
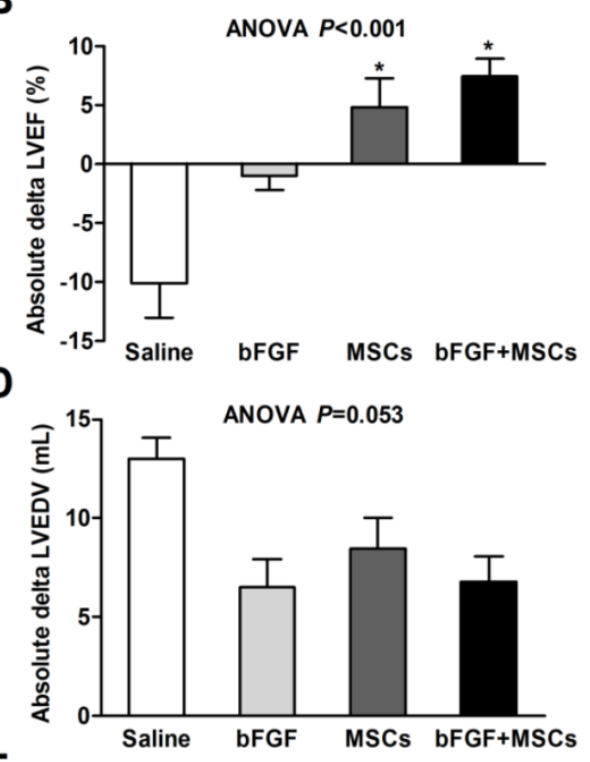

$\mathbf{F}$

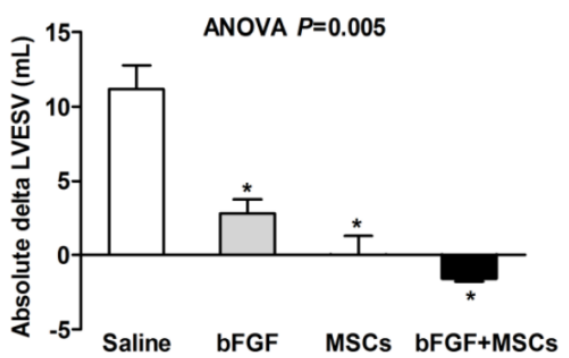

Figure 3. Retrograde infusion of combining basic fibroblast growth factor (bFGF) and mesenchymal stem cells (MSCs) restores cardiac function and prevents left ventricular (LV) remodeling. Echocardiographic analysis of (A) LV ejection fraction (LVEF), (C) LV end-diastolic volume (LVEDV), and (E) LV end-systolic volume (LVESV) before infusion and 4 weeks after treatment. The treatment effect (final minus pre-infusion parameters) was also evaluated (B, D, and $F$ ). (A) LVEF was improved in the bFGF+MSCs group, but remained unchanged in the bFGF group and MSCs group, and decreased in the saline group. The final LVEF and (B) the treatment effect were increased in the MSCs and bFGF+MSCs groups, to greater degree in the latter. (C) All groups exhibited increased LVEDV after infusion, with (D) mild attenuation in the intervention groups (not statistically significant). (E) LVESV remained stable in the intervention groups, but increased in the saline-treated group. At 4 weeks, the combined therapy presented lower LVESV in comparison to saline and bFGF alone groups. (F) The delta changes of LVESV demonstrated significant attenuation in the bFGF alone, MSCs alone, and bFGF+MSCs groups, to greatest degree in the combination group. Histograms (A, C, and E) show pre-infusion vs 4 weeks post-infusion values. Histograms represent mean \pm SEM. A, C, and E: $* p<0.05$ vs pre-infusion, paired $t$ test; $\dagger p<0.05$ vs saline, $\ddagger p<0.05$ vs bFGF, between-group repeated measures ANOVA. B, D, and F: $* p<0.05$ vs saline, 1-way ANOVA with Tukey post hoc test.

Table 1. Echocardiographic analysis. 


\begin{tabular}{|c|c|c|c|c|c|}
\hline Parameters & Saline $(n=3)$ & bFGF $(n=5)$ & $\operatorname{MSCs}(n=5)$ & bFGF+MSCs (n=5) & $p$ value (ANOVA) \\
\hline \multicolumn{6}{|l|}{ LVEDD (mm) } \\
\hline Baseline & $34.24 \pm 1.73$ & $35.62 \pm 0.65$ & $34.38 \pm 1.38$ & $32.90 \pm 2.28$ & 0.659 \\
\hline Pre-infusion & $36.92 \pm 0.46$ & $36.69 \pm 0.93$ & $35.43 \pm 1.70$ & $34.20 \pm 2.44$ & 0.696 \\
\hline Final & $40.07 \pm 0.57$ & $37.70 \pm 1.18$ & $36.28 \pm 1.97$ & $34.86 \pm 1.76$ & 0.275 \\
\hline Treatment effect & $3.14 \pm 0.67$ & $1.01 \pm 0.89$ & $0.85 \pm 0.93$ & $0.66 \pm 1.00$ & 0.351 \\
\hline \multicolumn{6}{|l|}{ LVESD (mm) } \\
\hline Baseline & $20.00 \pm 1.19$ & $20.99 \pm 1.68$ & $21.53 \pm 0.89$ & $19.67 \pm 1.29$ & 0.662 \\
\hline Pre-infusion & $22.33 \pm 0.73$ & $24.16 \pm 3.24$ & $23.20 \pm 1.31$ & $21.49 \pm 2.18$ & 0.815 \\
\hline Final & $26.50 \pm 0.36$ & $26.36 \pm 2.53$ & $24.08 \pm 1.63$ & $21.73 \pm 1.90$ & 0.273 \\
\hline Treatment effect & $4.17 \pm 0.91$ & $2.20 \pm 3.24$ & $0.88 \pm 0.74$ & $0.25 \pm 0.84$ & 0.124 \\
\hline \multicolumn{6}{|l|}{ FS (\%) } \\
\hline Baseline & $41.64 \pm 0.67$ & $41.17 \pm 4.02$ & $37.25 \pm 1.93$ & $40.09 \pm 1.42$ & 0.467 \\
\hline Pre-infusion & $39.54 \pm 1.24$ & $34.31 \pm 8.04$ & $34.45 \pm 2.26$ & $37.61 \pm 2.54$ & 0.411 \\
\hline Final & $33.86 \pm 0.38$ & $30.02 \pm 6.86$ & $33.52 \pm 3.06$ & $37.84 \pm 3.57$ & 0.587 \\
\hline Treatment effect & $-5.69 \pm 1.61$ & $-4.29 \pm 9.40$ & $-0.92 \pm 1.38$ & $0.23 \pm 2.34$ & 0.265 \\
\hline \multicolumn{6}{|l|}{ LVEDV (mL) } \\
\hline Baseline & $50.21 \pm 1.70$ & $52.48 \pm 3.21$ & $46.89 \pm 3.06$ & $44.09 \pm 7.14$ & 0.622 \\
\hline Pre-infusion & $58.07 \pm 1.59$ & $61.11 \pm 3.28$ & $50.48 \pm 3.85$ & $49.67 \pm 7.47$ & 0.291 \\
\hline Final & $71.04 \pm 1.53$ & $67.61 \pm 2.15$ & $58.92 \pm 5.34$ & $56.46 \pm 6.56$ & 0.273 \\
\hline Treatment effect & $12.98 \pm 1.11$ & $6.50 \pm 1.40$ & $8.44 \pm 1.58$ & $6.79 \pm 1.28$ & 0.053 \\
\hline \multicolumn{6}{|l|}{ LVESV (mL) } \\
\hline Baseline & $11.93 \pm 1.61$ & $13.18 \pm 1.91$ & $14.63 \pm 0.59$ & $12.40 \pm 1.87$ & 0.607 \\
\hline Pre-infusion & $18.12 \pm 0.88$ & $19.91 \pm 3.23$ & $17.99 \pm 0.73$ & $15.92 \pm 1.80$ & 0.729 \\
\hline Final & $29.32 \pm 1.63$ & $22.76 \pm 4.10$ & $18.04 \pm 1.37$ & $14.34 \pm 1.78$ & 0.003 \\
\hline Treatment effect & $11.20 \pm 1.57$ & $2.84 \pm 0.90$ & $0.05 \pm 1.26$ & $-1.57 \pm 0.23$ & 0.005 \\
\hline \multicolumn{6}{|l|}{ LVEF (\%) } \\
\hline Baseline & $76.40 \pm 2.44$ & $75.16 \pm 2.19$ & $68.40 \pm 1.84$ & $71.59 \pm 1.78$ & 0.066 \\
\hline Pre-infusion & $68.80 \pm 1.15$ & $67.60 \pm 4.12$ & $63.84 \pm 1.93$ & $67.17 \pm 1.43$ & 0.420 \\
\hline Final & $58.73 \pm 2.07$ & $66.59 \pm 5.07$ & $68.63 \pm 2.92$ & $74.61 \pm 1.14$ & 0.016 \\
\hline Treatment effect & $-10.07 \pm 2.94$ & $-1.01 \pm 1.17$ & $4.79 \pm 2.47$ & $7.43 \pm 1.51$ & $<0.001$ \\
\hline
\end{tabular}

Values are mean \pm SEM. Treatment effect indicates final minus pre-infusion parameters.

LVEDD indicates left ventricular end-diastolic dimension; LVESD, left ventricular end-systolic dimension; FS, fractional shortening; LVEDV, left ventricular end-diastolic volume; LVESV, left ventricular end-systolic volume; LVEF, left ventricular ejection fraction; bFGF, basic fibroblast growth factor; MSCs, mesenchymal stem cells.

\section{Combined therapy with bFGF and MSCs in- creases infarct thickness and alleviates myo- cardial fibrosis}

Gross morphologic analysis revealed midmural scars in the LV anterior and anteroseptal walls (Figure $4 \mathrm{~A})$. The average infarct wall thickness was increased in the bFGF+MSCs group $(8.2 \pm 0.2 \mathrm{~mm})$ compared with saline $(4.8 \pm 0.1 \mathrm{~mm})$, bFGF $(6.2 \pm 0.2 \mathrm{~mm})$, and MSCs $(6.6 \pm 0.1 \mathrm{~mm})$ groups (all $p<0.05$; Figure $4 \mathrm{~A}$ ). Additionally, the percentage of infarct wall to septal wall thickness was greatest in the bFGF+MSCs group (Figure 4A).

Masson's trichrome staining showed decreased collagen content in the infarct area in the MSCs alone group $(51.5 \pm 1.9 \%)$ and $b F G F+M S C s$ group $(47.3 \pm 1.6 \%)$, compared to the bFGF group $(59.8 \pm 2.9 \%)$ and saline group $(59.7 \pm 3.9 \%$ ) (all $p<0.05$; Figure $4 \mathrm{~B})$, to greater degree in the combination group. Reduced border zone collagen content was observed in the MSCs alone group $(38.1 \pm 2.3 \% ; p<0.05)$ and bFGF+MSCs group $(36.6 \pm 1.8 \% ; p<0.05)$ compared to saline $(47.7 \pm 1.7 \%)$ (Figure $4 \mathrm{~B})$. Interestingly, more myocardium islets were detected within the infarct scars of the combination-treated animals.

\section{Combined therapy with bFGF and MSCs stimulates new blood vessel formation and reduces apoptosis}

Capillary and arteriole density was determined (respectively by FVIII and a-SMA staining) in the infarct border zones. The number of $\mathrm{FVIII}^{+}$cells was markedly greater in the bFGF+MSCs group $\left(97.1 \pm 8.3 / \mathrm{mm}^{2}\right) \quad$ compared to the saline $\left(40.0 \pm 5.3 / \mathrm{mm}^{2} ; \quad p<0.001\right)$, bFGF $\left(60.0 \pm 5.2 / \mathrm{mm}^{2}\right.$; $p<0.01)$, and MSCs $\left(54.3 \pm 5.4 / \mathrm{mm}^{2} ; p<0.001\right)$ groups (Figure 5A). The arteriolar density was significantly greater in the bFGF+MSCs group $\left(35.7 \pm 3.2 / \mathrm{mm}^{2}\right)$ compared to the saline $\left(15.0 \pm 2.4 / \mathrm{mm}^{2} ; p<0.05\right)$ and MSCs (23.6 $\left.\pm 2.4 / \mathrm{mm}^{2} ; p<0.05\right)$ groups (Figure $5 \mathrm{~B}$ ).

Apoptosis was assessed by the proportion of TUNEL $^{+}$cells in the infarct border zone, which was significantly decreased in the animals treated with bFGF+MSCs $(8.2 \pm 0.9 \%)$ compared to saline $(14.6 \pm 0.9 \% ; p<0.01)$ (Figure $5 \mathrm{C}$ ), but similar to bFGF alone $(12.5 \pm 1.7 \%)$ and MSCs alone $(12.3 \pm 0.5 \%)$.

\section{bFGF enhances engraftment and differentia- tion of transplanted MSCs}

To address whether bFGF improved MSCs engraftment and survival, the transplanted cells were 
labeled with EGFP and observed in the myocardium 4 weeks after delivery. The $\mathrm{EGFP}^{+}$cells localized primarily in the infarct and border zones (Figure 6A-6D). The number of $\mathrm{EGFP}^{+}$cells was 1-fold higher in the bFGF+MSCs group $\left(83.9 \pm 9.6 / \mathrm{mm}^{2}\right)$ compared to the MSCs alone group $\left(42.3 \pm 7.4 / \mathrm{mm}^{2} ; p<0.05\right)$ (Figure $6 \mathrm{E})$.

Surviving MSCs mainly differentiated into vascular structures, indicated by positive staining with FVIII (Figure 6A) and a-SMA (Figure 6B). Compared to the MSCs group, the bFGF+MSCs group exhibited more $\mathrm{FVIII}^{+}\left(30.2 \pm 3.1 / \mathrm{mm}^{2}\right.$ vs $\left.8.9 \pm 1.8 / \mathrm{mm}^{2} ; p<0.001\right)$ and $\mathrm{a}_{-} \mathrm{SMA}^{+}\left(9.9 \pm 0.7 / \mathrm{mm}^{2}\right.$ vs $\left.3.9 \pm 0.4 / \mathrm{mm}^{2} ; p<0.05\right)$ cells (Figure 6F). Moreover, the coexpression of TnI and $\mathrm{MHC}$ in $\mathrm{EGFP}^{+}$cells indicated some MSCs had differentiated into a cardiomyocyte phenotype (Figure $6 \mathrm{C}$ and $6 \mathrm{D}$ ), although no significant difference was found between the two groups (Figure 6F). Collectively, bFGF appears to enhance the differentiation capability of MSCs under both hypoxic conditions (Figure 2C) and infarction circumstances (Figure 6).

\section{A}
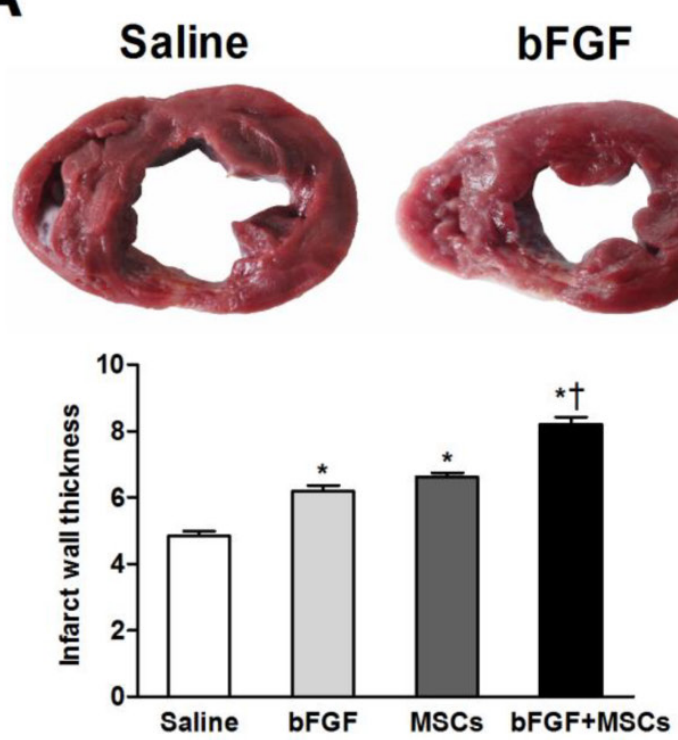

B

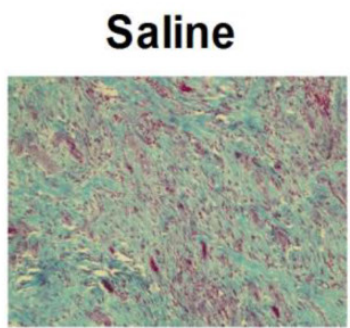

\section{bFGF}

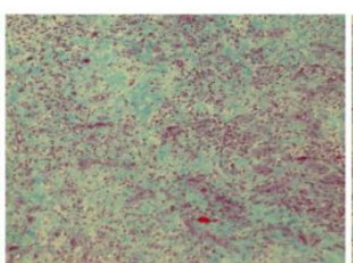

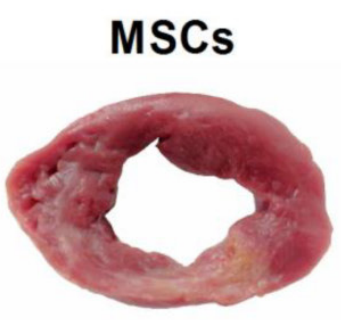

bFGF+MSCs
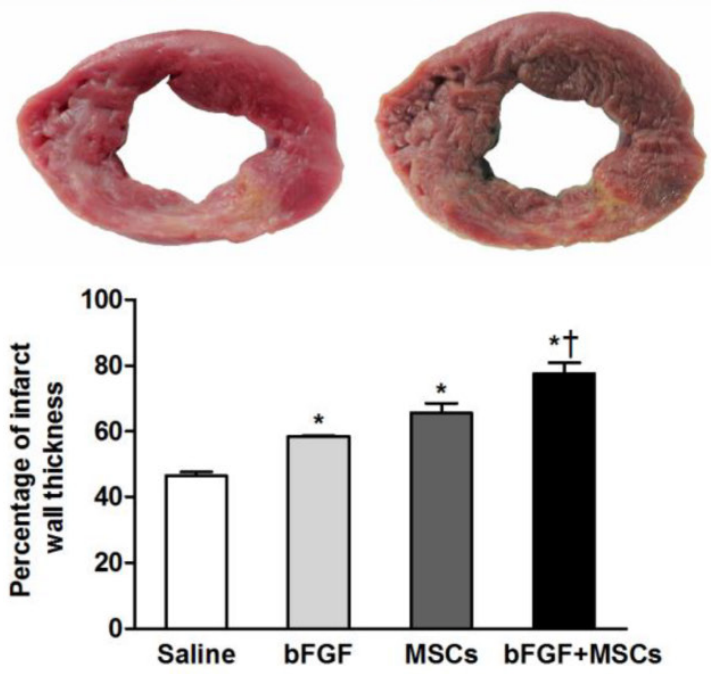

MSCs

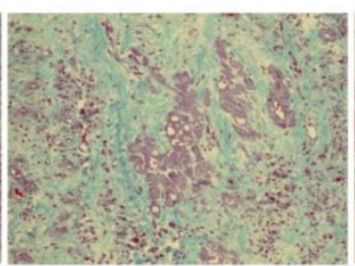

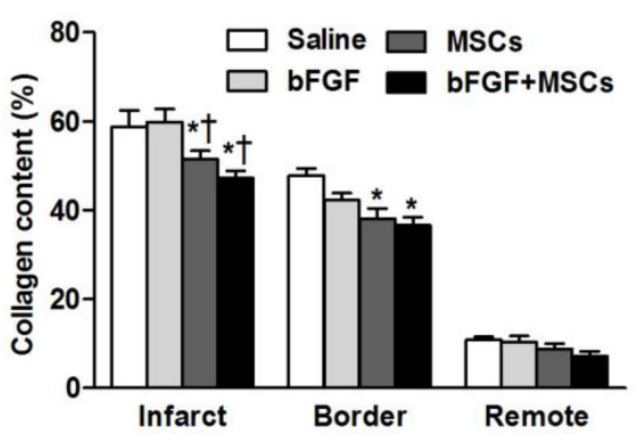

Figure 4. Combined administration of basic fibroblast growth factor (bFGF) and mesenchymal stem cells (MSCs) increases infarct wall thickness and reduces myocardial fibrosis. (A) Post-mortem morphometry revealed midmural scars in the left ventricular wall. Coronary venous infusion of bFGFs+MSCs increased infarct wall thickness compared to other groups. The percentage of infarct wall thickness to septal wall thickness was greatest in the bFGF+MSCs group. (B) Masson's trichrome staining showed decreased collagen content in the MSCs and bFGF+MSCs groups both in the infarct and border zones. Scale bar: $100 \mu m$. Histograms represent mean $\pm S E M$. A: $* p<0.05$ vs saline; $\nmid p<0.05$ vs bFGF and MSCs groups. B: ${ }^{*} p<0.05$ vs saline; $\dagger p<0.05$ vs bFGF. 
A

Saline

bFGF

MSCs

bFGF+MSCs
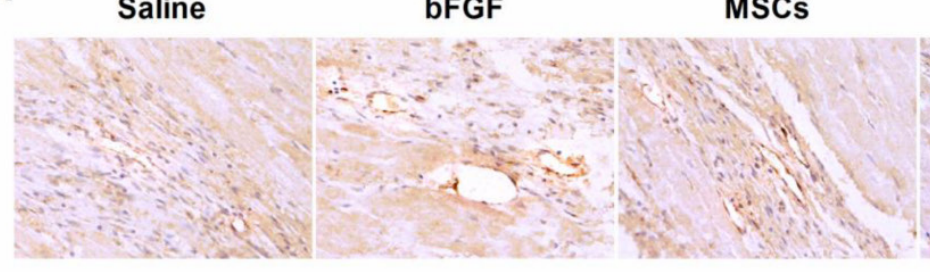

bFGF+MSCs

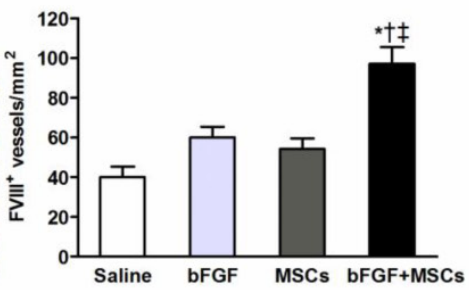

B

Saline

bFGF

MSCs
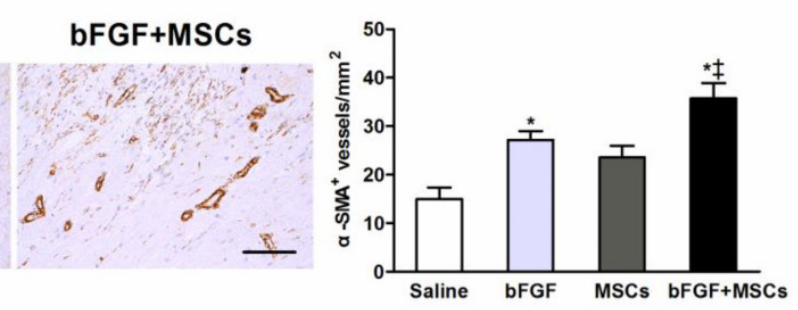

C

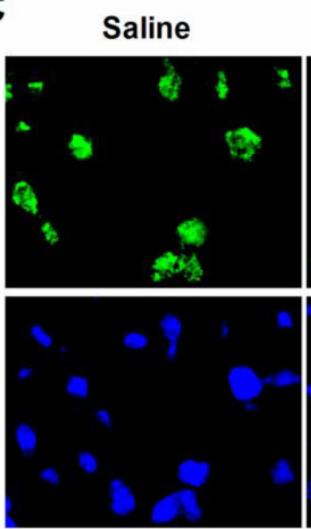

bFGF

MSCs

bFGF+MSCs
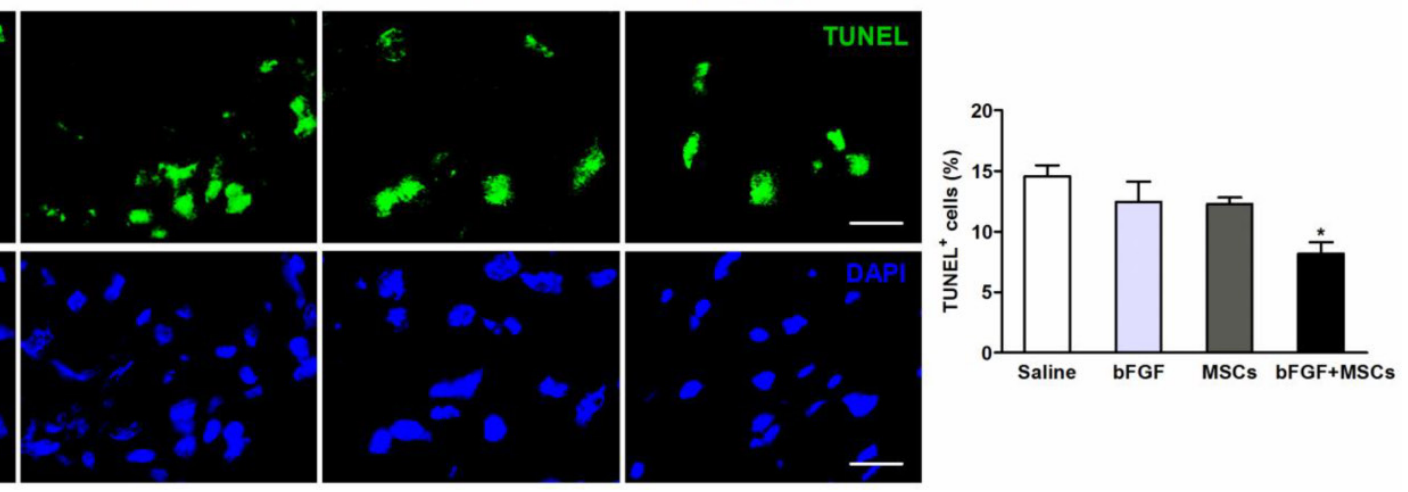

Figure 5. Combined administration of basic fibroblast growth factor (bFGF) and mesenchymal stem cells (MSCs) induces neovascularization and inhibits apoptosis. Representative micrographs demonstrated formation of (A) new capillaries and (B) arterioles in the infarct border region after treatment. Quantitative analysis indicated markedly greater (A) capillary density (evidenced by factor VIII-related antigen [FVIII] staining) and (B) arteriole density (evidenced by $\alpha$-smooth muscle actin [ $\alpha$-SMA] staining) in the bFGF+MSCs group. (C) Representative images of terminal deoxynucleotidyl transferase dUTP nick end labeling (TUNEL)-positive cells in the border region of the infarct myocardium. Cell nuclei were counterstained with 4,6-diamidino-2-phenylindole (DAPI). The proportion of TUNEL+ cells was significantly decreased in the bFGF+MSCs group. Scale bars: $50 \mu \mathrm{m}$ (A); $100 \mu \mathrm{m}$ (B); $20 \mu \mathrm{m}$ (C). Histograms represent mean $\pm S E M$. $* p<0.05$ vs saline; $\nmid p<0.05$ vs bFGF; $\neq p<0.05$ vs MSCs. All with 1 -way ANOVA by Tukey post hoc test.

\section{Discussion}

In the present study, we demonstrate the coronary vein is a feasible and safe route for delivery of bFGF and MSCs. bFGF augments the engraftment and differentiation of transplanted MSCs in vivo. Retrograde infusion of MSCs with concomitant bFGF results in enhanced cardiac function recovery and prevention of LV remodeling post-MI. Increased neovascularization and reduced apoptosis are also observed. Taken together, these data indicate that concomitant bFGF and MSCs via coronary vein infusion confers improved therapeutic efficacy in a canine MI model, thereby representing a promising future strategy for cardiac repair after ischemic insult.

\section{MSCs as a Candidate for Cardiac Regeneration Therapy}

In the past decade, numerous studies have been seeking an ideal stem-cell type suitable for cardiac repair. Indeed, MSCs are a desirable agent for myo- cardial repair because of preparatory ease, advantageous immunomodulatory properties, and favorable biological effects [2]. On the basis of encouraging pre-clinical studies [3-5], proof-of-concept clinical trials have been initiated for both acute MI [7] and ischemic cardiomyopathy [6] and demonstrated that MSCs therapy improved cardiac performance, inhibited LV remodeling, and reduced scar size, with a remarkable safety profile. Recently, studies have focused upon the candidacy of resident cardiac stem cells (CSCs) for cardiac repair, due to their cardiac source and potent cardiomyogenic potential [23, 24]. In large animal models, CSCs injection improved LV function and reduced scar size with evidence of cardiomyocyte and vascular regeneration [25]. However, recent clinical trials (SCIPIO [26] and CADUCEUS [27]) revealed inconsistent results in terms of LVEF, symptoms, or quality of life after intracoronary CSCs administration in patients with ischemic cardiomyopathy. The CSCs ready for transplantation must be autologous, and require several weeks of in vitro ex- 
pansion, factors limiting their use in the acute MI setting [23]. It is clinically impractical to perform endomyocardial biopsy immediately after infarction.

A
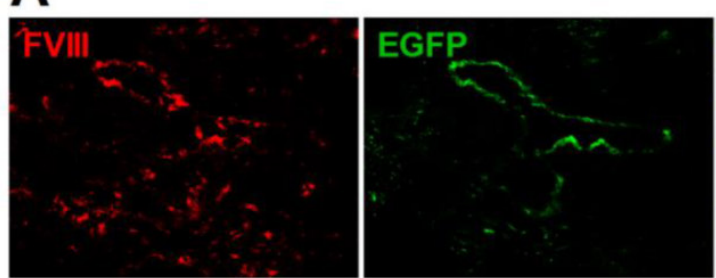

B
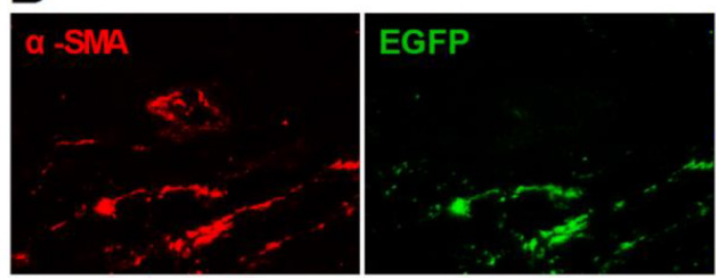

C
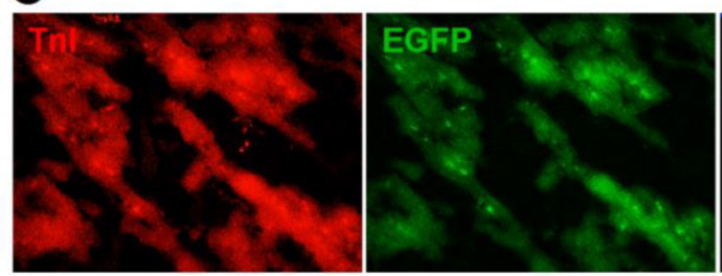

D
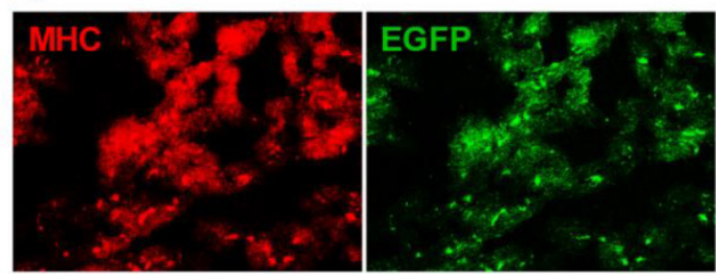

E

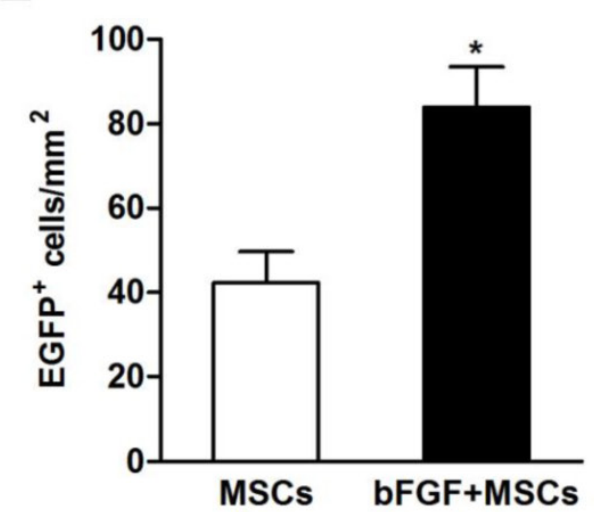

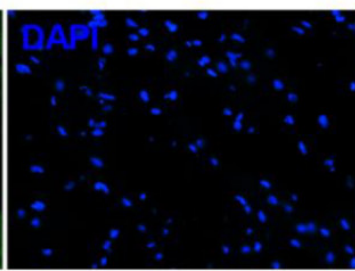
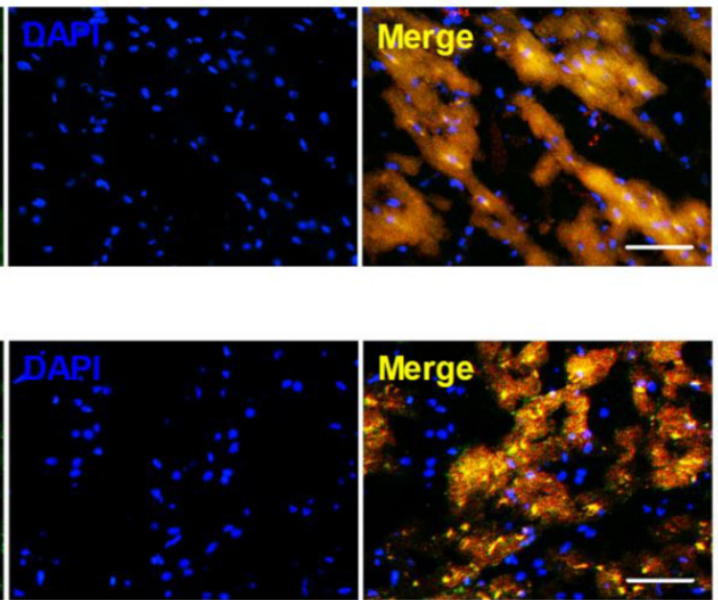

Therefore, generic allogenic MSCs remain the most accessible cell product for cardiac regeneration therapy at the current time.
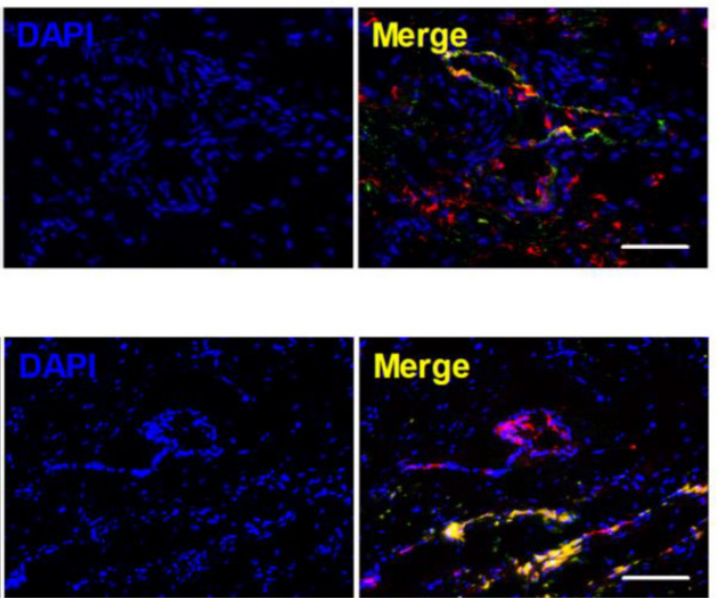

$\mathbf{F}$

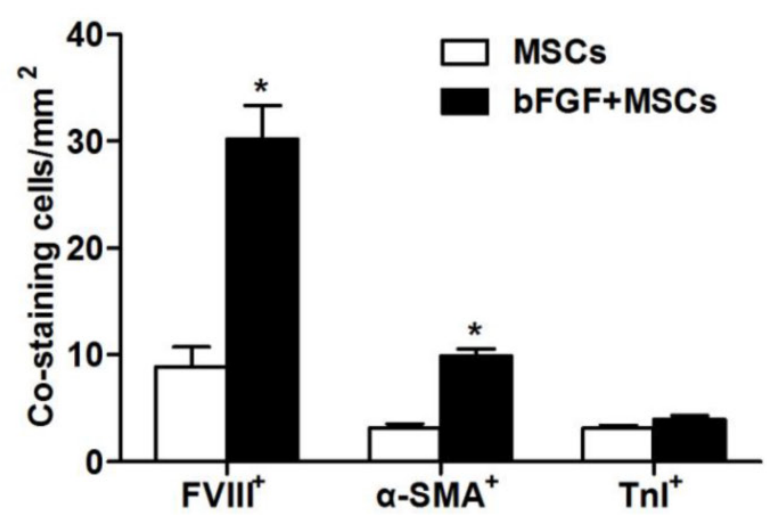

Figure 6. Retrograde infusion of basic fibroblast growth factor (bFGF) enhances mesenchymal stem cells (MSCs) engraftment and differentiation. Representative immunofluorescence images demonstrated coexpression of (A) factor VIII-related antigen (FVIII), (B) $\alpha$-smooth muscle actin ( $\alpha$-SMA), (C) cardiac troponin I (Tnl), and (D) cardiac myosin heavy chain (MHC) in enhanced green fluorescence protein (EGFP) positive cells in the infarct region. Nuclei were stained with 4,6-diamidino-2-phenylindole (DAPI). (E) The number of EGFP+ cells was 1-fold higher in the bFGF+MSCs group than in the MSCs group. (F) The number of labeled cells costained with FVIII and a-SMA was significantly greater in the animals treated with bFGF+MSCs $(n=3)$ compared with MSCs ( $n=3)$, indicating enhanced neovascularization by combined therapy. The number of EGFP+ cells coexpressing Tnl was not statistically different between the two groups. Scale bars: $100 \mu m(A, C$ and D); $200 \mu m(B)$. Histograms represent mean \pm SEM. $* p<0.05$ vs MSCs, unpaired Student $t$ test. 


\section{Combined efficacy of bFGF and MSCs}

Despite the beneficial effects of MSCs, the clinical application is impeded by poor engraftment and survival of transplanted cells in the host myocardium. In animal studies, the average retention rate was less than $10 \%$ in the acute phase of MI $[19,28]$, and only $5 \%$ of implanted MSCs survived for 14 days in the infarcted myocardium regardless of the delivery route [16]. Targeted homing of MSCs towards injured tissue is modulated by several growth factors [11]. Our vitro studies demonstrated increased MSCs migratory activity during coculture with bFGF, in consistent fashion with previous studies [13]. Therefore, it is plausible the combination of MSCs with bFGF might facilitate increased cellular engraftment and therapeutic potency. Takehara et al. demonstrated controlled bFGF delivery promoted human cardiosphere-derived cell engraftment with improved ventricular function [29]. In parallel, we now demonstrate bFGF cotreatment augments MSCs retention within the host myocardium with increased LVEF.

LV remodeling is characterized by wall thinning and chamber dilatation and leads to worsen cardiac function. Previous studies have shown administration of stem cells can increase infarct wall thickness and improved ventricular remodeling, probably by alleviating myocardial fibrosis and reducing apoptosis. Ye et al. [30] used a swine MI model and presented that intramyocardial injection of thymosin $\beta 4$ and MSCs significantly increased the percentage of infarct wall thickness to septal wall thickness compared to control group ( $50 \%$ vs. $25 \%, p<0.05)$. Houtgraaf et al. [31] used a sheep MI model and demonstrated intracoronary infusion of mesenchymal precursor cells significantly enhanced the average infarct wall thickness compared to control group $(8.0 \pm 0.3 \mathrm{~mm}$ vs. $6.4 \pm 0.2 \mathrm{~mm}, p<0.001)$. In consistent with these studies, our results showed the infarct wall thickness was increased in the MSCs group $(6.6 \pm 0.1 \mathrm{~mm})$ compared with saline $(4.8 \pm 0.1 \mathrm{~mm}, p<0.05)$, and bFGF+MSCs group exhibited more obvious wall thickness improvement $(8.2 \pm 0.2 \mathrm{~mm}, \quad p<0.05$ vs all the other groups). The combination group also exhibited alleviated myocardial fibrosis and apoptosis. These results can explain the significant improvement of infarct wall thickness in the combined treatment group.

To further clarify the underlying mechanism of the beneficial effects of combined treatment, the fate of injected MSCs was monitored by dual labeling with EGFP and cardiac-specific markers. We found the engrafted MSCs primarily differentiated into endothelial cells and smooth muscle cells, as indicated by coexpression of EGFP with FVIII and a-SMA, suggesting vascular regeneration. This phenomenon was more evident in the combination bFGF+MSCs group. Differentiation of MSCs into cardiomyocytes was also observed, but was not enhanced by bFGF. In fact, the differentiation capacity of transplanted MSCs in the ischemic heart is influenced by many factors and the study results are conflicting. In a swine model of chronic ischemic cardiomyopathy, Quevedo et al. [8] reported the capacity of allogeneic MSCs to engraft and differentiate into cardiomyocytes, smooth muscle cells and endothelial cells. In contrast, in a canine chronic ischemic model, Silva et al. [4] showed that MSCs can differentiate into cells with a vascular phenotype, but few cardiomyocytes differentiated from MSCs were detected. Actually, the average retention rate of MSCs was low in the acute phase of MI, and the morphology and mobility of transplanted MSCs were also impaired in the infarcted myocardial environment [32]. It thus appears that the overall differentiation ratio of MSCs into cardiomyocytes was relatively low. Therefore, in our study, although retrograde infusion of bFGF significantly enhanced MSCs engraftment in the myocardium, the number of cardiomyocytes differentiated from MSCs was modestly increased by bFGF, and the engrafted MSCs mainly differentiated into vascular cell lineage, which is consistent with previous studies.

The harsh ischemic microenvironment, characterized by oxidative stress, inflammation, and limited blood supply, hinders the survival of transplanted MSCs [33]. The bFGF involved in the MSCs secretome stimulates angiogenesis, and improves the ischemic surroundings [34]. Although bFGF alone promoted new arteriolar formation, LV functional improvements could not be reached without the MSCs. Our study demonstrated concomitant bFGF infusion with MSCs ameliorated myocardial fibrosis, increased capillary/arteriolar density, and reduced apoptosis, effects that may stem from bFGF-induced angiogenesis and MSCs differentiation. Together, these observations support the notion that the interaction between bFGF and MSCs synergistically optimized measured outcomes.

\section{Mode of delivery}

Heretofore, the optimal delivery technique of candidate cells to the heart remains undetermined. An ideal system must attain a desired cell population, and facilitate targeted and homogeneous cell dissemination within the myocardium with low risk of complications. Direct surgical injection provides a great cell population per unit area, but is limited by non-uniform spatial distribution [17]. Intracoronary administration is easy to perform, but immediate cell retention is low due to rapid wash out [19]. Additionally, this approach carries a high risk of micro- 
vascular occlusion when large cells, such as MSCs, are infused [18]. Currently available transendocardial injection enables cell delivery into targeted myocardium [35]. However, this technique may create cell clumps without homogeneous distribution, and myocardial perforation and arrhythmias must be monitored $[2,18]$.

In contrast, the retrograde route is an attractive mode of delivery because it employs the non-diseased coronary veins, which parallel the arterial system, for selective delivery to ischemic myocardium [36]. Increased tissue penetration using this method is probably due to improved access to low-pressure capillary beds, and less washout of cells in the presence of reduced anterograde blood flow [36]. Yokoyama and colleagues [37] demonstrated $>90 \%$ of labeled mononuclear cells remained in the infarct region post coronary vein infusion. Suzuki and colleagues [22] reported retrograde delivery of skeletal muscle precursor cells provided wide dissemination throughout the left ventricular free wall, with minimal myocardial damage. Our study demonstrated the balloon position was consistent with the occlusive LAD site (Figure 1). The delivery site was within the targeted infarct zone. As a result, the transplanted MSCs engrafted primarily within the desired region downstream of the occluded LAD.

To maximize the beneficial effects of bFGF upon MSCs, we utilized for the first time a retrograde approach, for the concomitant infusion of bFGF and MSCs. Previous study showed coronary vein infusion of MSCs prevents cardiac dysfunction in a chronic pig MI model, but with limited cell retention and differentiation [38]. In our study, we employed a proximal balloon occlusion, achieving a bFGF reservoir, which exerted sustained effect upon MSCs. Our results revealed combination therapy with bFGF and MSCs augmented cell engraftment and differentiation, with enhanced cardiac structural and functional performance.

\section{Limitations}

The canine model employed in our study might limit interpretation of cardiac functional data, because dogs develop numerous collateral circulations in ischemic conditions. We attempted to decrease this physiologic effect as much as possible by diagonal branches ligation whenever appropriate. Although each group's sample size is small, statistical significances were observed between treatment groups in both echocardiographic parameters and histologic findings. More advanced modalities such as 3D echocardiography and cardiac magnetic resonance imaging should be considered for more accurate assessment of cardiac morphology and function, espe- cially when sample size is small. This study used an OTW balloon catheter to provide proximal occlusion for cell infiltration, but this technique would not overcome cell washout due to venovenous communications. A double-balloon catheter design is promising [19]. Free exogenous bFGF might have limited efficacy due to short half-life in the body. In consideration of this question, we used retrograde coronary venous approach to achieve high local concentration and more uniform delivery. Further studies using bFGF modification technique (eg. nanoparticles) to increase the half-life of bFGF are needed. The coupling of exogenous MSCs with native cardiomyocytes can better identify the engraftment and differentiation of transplanted MSCs within host myocardium, and have been investigated by several previous studies [3, 39], although it is not the focus of our study. Importantly, the signal pathway involved in the interplay between bFGF and MSCs for cardiac repair remains unclear, and warrants rigorous investigation.

\section{Supplementary Materials}

Supplementary Methods. http://www.thno.org/v05p0995s1.pdf

\section{Conclusions}

The present study demonstrated retrograde coronary venous bFGF infusion enhances the engraftment and differentiation capacity of transplanted MSCs in response to MI injury. Co-administration of bFGF and MSCs significantly improves cardiac function and ameliorates adverse remodeling, which is accompanied by increased neovascularization and reduced apoptosis. Taken together, our findings suggest a novel combined therapy via retrograde infusion may represent a promising strategy for cardiac repair after ischemic injury.

\section{Abbreviations}

a-SMA: a-smooth muscle actin; AIV: anterior interventricular vein; ANOVA: analysis of variance; bFGF: basic fibroblast growth factor; CSCs: cardiac stem cells; EGFP: enhanced green fluorescence protein; FS: fractional shortening; IGF-1: insulin-like growth factor; LAD: left anterior descending; LV: left ventricular; LVEDD: left ventricular end-diastolic diameter; LVEDV: left ventricular end-diastolic volume; LVEF: LV ejection fraction; LVESD: left ventricular end-systolic diameter; LVESV: left ventricular end-systolic volume; MHC: myosin heavy chain; MI: myocardial infarction; MSCs: mesenchymal stem cells; OTW: over-the-wire; TnI: troponin I; VEGF: vascular endothelial growth factor. 


\section{Acknowledgements}

This work was supported by grants from the National Natural Science Foundation of China (No. 81070166 and No. 81270284) and the Beijing Natural Science Foundation (7141003).

\section{Competing Interests}

The authors have declared that no competing interest exists.

\section{References}

1. Behfar A, Crespo-Diaz R, Terzic A, Gersh BJ. Cell therapy for cardiac repair--lessons from clinical trials. Nat Rev Cardiol. 2014; 11: 232-46. doi:10.1038/nrcardio.2014.9.

2. Williams AR, Hare JM. Mesenchymal stem cells: biology, pathophysiology, translational findings, and therapeutic implications for cardiac disease. Circ Res. 2011; 109: 923-40. doi:10.1161/CIRCRESAHA.111.243147.

3. Hatzistergos KE, Quevedo H, Oskouei BN, Hu Q, Feigenbaum GS, Margitich IS, et al. Bone marrow mesenchymal stem cells stimulate cardiac stem cell proliferation and differentiation. Circ Res. 2010; 107: 913-22. doi:10.1161/CIRCRESAHA.110.222703.

4. Silva GV, Litovsky S, Assad JA, Sousa AL, Martin BJ, Vela D, et al. Mesenchymal stem cells differentiate into an endothelial phenotype, enhance vascular density, and improve heart function in a canine chronic ischemia model. Circulation. 2005; 111: 150-6. doi:10.1161/01.CIR.0000151812.86142.45

5. Tao B, Cui M, Wang C, Ma S, Wu F, Yi F, et al. Percutaneous intramyocardial delivery of mesenchymal stem cells induces superior improvement in regional left ventricular function compared with bone marrow mononuclear cells in porcine myocardial infarcted heart. Theranostics. 2015; 5: 196-205. doi:10.7150/thno.7976.

6. Heldman AW, DiFede DL, Fishman JE, Zambrano JP, Trachtenberg BH, Karantalis V, et al. Transendocardial mesenchymal stem cells and mononuclear bone marrow cells for ischemic cardiomyopathy: the TAC-HFT randomized trial. JAMA. 2014; 311: 62-73. doi:10.1001/jama.2013.282909.

7. Hare JM, Traverse JH, Henry TD, Dib N, Strumpf RK, Schulman SP, et al. A randomized, double-blind, placebo-controlled, dose-escalation study of intravenous adult human mesenchymal stem cells (prochymal) after acute myocardial infarction. J Am Coll Cardiol. 2009; 54: 2277-86. doi:10.1016/j.jacc.2009.06.055

8. Quevedo HC, Hatzistergos KE, Oskouei BN, Feigenbaum GS, Rodriguez JE, Valdes D, et al. Allogeneic mesenchymal stem cells restore cardiac function in chronic ischemic cardiomyopathy via trilineage differentiating capacity. Proc Natl Acad Sci U S A. 2009; 106: 14022-7. doi:10.1073/pnas.0903201106.

9. Gnecchi M, Zhang Z, Ni A, Dzau VJ. Paracrine mechanisms in adult stem cell signaling and therapy. Circ Res. 2008; 103: 1204-19. doi:10.1161/CIRCRESAHA.108.176826.

10. Terrovitis JV, Smith RR, Marban E. Assessment and optimization of cell engraftment after transplantation into the heart. Circ Res. 2010; 106: 479-94. doi:10.1161/CIRCRESAHA.109.208991.

11. Yagi H, Soto-Gutierrez A, Parekkadan B, Kitagawa Y, Tompkins RG, Kobayashi N, et al. Mesenchymal stem cells: Mechanisms of immunomodulation and homing. Cell Transplant. 2010; 19: 667-79. doi:10.3727/096368910X508762.

12. Beenken A, Mohammadi M. The FGF family: biology, pathophysiology and therapy. Nat Rev Drug Discov. 2009; 8: 235-53. doi:10.1038/nrd2792.

13. Schmidt A, Ladage D, Schinkothe T, Klausmann U, Ulrichs C, Klinz FJ, et al. Basic fibroblast growth factor controls migration in human mesenchymal stem cells. Stem Cells. 2006; 24: 1750-8. doi:10.1634/stemcells.2005-0191.

14. Song H, Kwon K, Lim S, Kang SM, Ko YG, Xu Z, et al. Transfection of mesenchymal stem cells with the FGF-2 gene improves their survival under hypoxic conditions. Mol Cells. 2005; 19: 402-7.

15. Dib N Menasche P, Bartunek JJ, Zeiher AM, Terzic A, Chronos NA, et al. Recommendations for successful training on methods of delivery of biologics for cardiac regeneration: a report of the International Society for Cardiovascular Translational Research. JACC Cardiovasc Interv. 2010; 3: 265-75. doi:10.1016/j.jcin.2009.12.013.

16. Freyman T, Polin G, Osman H, Crary J, Lu M, Cheng L, et al. A quantitative, randomized study evaluating three methods of mesenchymal stem cell delivery following myocardial infarction. Eur Heart J. 2006; 27: 1114-22. doi:10.1093/eurheartj/ehi818.

17. Grossman PM, Han Z, Palasis M, Barry JJ, Lederman RJ. Incomplete retention after direct myocardial injection. Catheter Cardiovasc Interv. 2002; 55: 392-7. doi:10.1002/ccd.10136.

18. Sanganalmath SK, Bolli R. Cell therapy for heart failure: a comprehensive overview of experimental and clinical studies, current challenges, and future directions. Circ Res. 2013; 113: 810-34. doi:10.1161/CIRCRESAHA.113.300219.

19. Hou D, Youssef EA, Brinton TJ, Zhang P, Rogers P, Price ET, et al. Radiolabeled cell distribution after intramyocardial, intracoronary, and interstitial retrograde coronary venous delivery: implications for current $\begin{array}{llll}\text { clinical trials. } & \text { Circulation. 2005; 112: }\end{array}$ doi:10.1161/CIRCULATIONAHA.104.526749.

20. Fearon WF, Ikeno F, Bailey LR, Hiatt BL, Herity NA, Carter AJ, et al. Evaluation of high-pressure retrograde coronary venous delivery of FGF-2 protein. Catheter Cardiovasc Interv. 2004; 61: 422-8. doi:10.1002/ccd.10790.

21. Wang X, Nie S-P, Zhen L, Miao H-T, Wu X-X, Ren H-M, et al. Retrograde coronary venous infusion provides targeted cell engraftment into infarcted

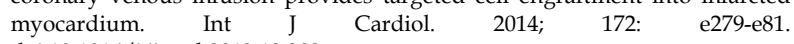
doi:10.1016/j.ijcard.2013.12.202.

22. Suzuki K, Murtuza B, Fukushima S, Smolenski RT, Varela-Carver A, Coppen $\mathrm{SR}$, et al. Targeted cell delivery into infarcted rat hearts by retrograde intracoronary infusion: distribution, dynamics, and influence on cardiac function. Circulation. 2004; 110 II225-30. doi:10.1161/01.CIR.0000138191.11580.e3.

23. Pfister O, Della Verde G, Liao R, Kuster GM. Regenerative therapy for cardiovascular disease. Transl Res. 2014; 163: 307-20. doi:10.1016/j.trsl.2013.12.005.

24. Garbern JC, Lee RT. Cardiac stem cell therapy and the promise of heart regeneration. Cell Stem Cell. 2013; 12: 689-98. doi:10.1016/j.stem.2013.05.008

25. Bolli R, Tang XL, Sanganalmath SK, Rimoldi O, Mosna F, Abdel-Latif A, et al. Intracoronary delivery of autologous cardiac stem cells improves cardiac function in a porcine model of chronic ischemic cardiomyopathy. Circulation. 2013; 128: 122-31. doi:10.1161/CIRCULATIONAHA.112.001075.

26. Bolli R, Chugh AR, D'Amario D, Loughran JH, Stoddard MF, Ikram S, et al. Cardiac stem cells in patients with ischaemic cardiomyopathy (SCIPIO): initial results of a randomised phase 1 trial. Lancet. 2011; 378: 1847-57. doi:10.1016/S0140-6736(11)61590-0.

27. Makkar RR, Smith RR, Cheng K, Malliaras K, Thomson LE, Berman D, et al. Intracoronary cardiosphere-derived cells for heart regeneration after myocardial infarction (CADUCEUS): a prospective, randomised phase 1 trial. Lancet. 2012; 379: 895-904. doi:10.1016/S0140-6736(12)60195-0.

28. Tang YL, Tang Y, Zhang YC, Qian K, Shen L, Phillips MI. Improved graft mesenchymal stem cell survival in ischemic heart with a hypoxia-regulated heme oxygenase-1 vector. J Am Coll Cardiol. 2005; 46: 1339-50. doi:10.1016/j.jacc.2005.05.079.

29. Takehara N, Tsutsumi $\mathrm{Y}$, Tateishi $\mathrm{K}$, Ogata T, Tanaka H, Ueyama T, et al. Controlled delivery of basic fibroblast growth factor promotes human cardiosphere-derived cell engraftment to enhance cardiac repair for chronic myocardial infarction. J Am Coll Cardiol. 2008; 52: 1858-65. doi:10.1016/j.jacc.2008.06.052

30. Ye L, Zhang P, Duval S, Su L, Xiong Q, Zhang J. Thymosin beta4 increases the potency of transplanted mesenchymal stem cells for myocardial repair. Circulation. 2013; 128: S32-41. doi:10.1161/CIRCULATIONAHA.112.000025.

31. Houtgraaf JH, de Jong R, Kazemi K, de Groot D, van der Spoel TI, Arslan F, et al. Intracoronary infusion of allogeneic mesenchymal precursor cells directly after experimental acute myocardial infarction reduces infarct size, abrogates adverse remodeling, and improves cardiac function. Circ Res. 2013; 113: 153-66. doi:10.1161/CIRCRESAHA.112.300730

32. Liechty KW, MacKenzie TC, Shaaban AF, Radu A, Moseley AM, Deans R, et al. Human mesenchymal stem cells engraft and demonstrate site-specific differentiation after in utero transplantation in sheep. Nat Med. 2000; 6: 1282-6. doi:10.1038/81395

33. Rodrigues M, Griffith LG, Wells A. Growth factor regulation of proliferation and survival of multipotential stromal cells. Stem Cell Res Ther. 2010; 1: 32. doi:10.1186/scrt32.

34. Ranganath SH, Levy O, Inamdar MS, Karp JM. Harnessing the mesenchymal stem cell secretome for the treatment of cardiovascular disease. Cell Stem Cell. 2012: 10: 244-58 doi:10.1016/j.stem 2012.02.005.

35. Vrtovec B, Poglajen G, Lezaic L, Sever M, Socan A, Domanovic D, et al. Comparison of transendocardial and intracoronary CD34+ cell transplantation in patients with nonischemic dilated cardiomyopathy. Circulation. 2013; 128: S42-9. doi:10.1161/CIRCULATIONAHA.112.000230.

36. Jain AK, Smith EJ, Rothman MT. The coronary venous system: an alternative route of access to the myocardium. J Invasive Cardiol. 2006; 18: 563-8.

37. Yokoyama S, Fukuda N, Li Y, Hagikura K, Takayama T, Kunimoto S, et al. A strategy of retrograde injection of bone marrow mononuclear cells into the myocardium for the treatment of ischemic heart disease. J Mol Cell Cardiol. 2006; 40: 24-34. doi:10.1016/j.yjmcc.2005.06.008.

38. Sato T, Iso Y, Uyama T, Kawachi K, Wakabayashi K, Omori Y, et al. Coronary vein infusion of multipotent stromal cells from bone marrow preserves cardiac function in swine ischemic cardiomyopathy via enhanced neovascularization. Lab Invest. 2011; 91: 553-64 doi:10.1038/labinvest.2010.202.

39. Behfar A, Yamada S, Crespo-Diaz R, Nesbitt JJ, Rowe LA, Perez-Terzic C, et al. Guided cardiopoiesis enhances therapeutic benefit of bone marrow human mesenchymal stem cells in chronic myocardial infarction. J Am Coll Cardiol. 2010; 56: 721-34. doi:10.1016/j.jacc.2010.03.066 